RELATO DE CASO

\title{
Correção cirúrgica de aneurismas saculares de fístula arteriovenosa para hemodiálise utilizando a técnica de aneurismorrafia
}

\author{
Surgical repair of saccular aneurysms of an arteriovenous fistula for hemodialysis using \\ aneurismorraphy technique
}

Ricardo Wagner da Costa Moreira', David Domingos Rosado Carrilho², Kellen Micheline Alves Henrique Costa ${ }^{3}$, Rafaela Brito Bezerra Pinheiro ${ }^{4}$

\begin{abstract}
Resumo
Uma das complicações mais frequentes da fístula arteriovenosa (FAV) para hemodiálise é o desenvolvimento de um aneurisma. A formação de aneurismas geralmente decorre de um enfraquecimento da parede venosa devido às repetidas punções, e sua rotura causa hemorragia intensa que pode levar à morte. Descrevemos o caso de uma paciente com dois aneurismas saculares de FAV, tratados através de aneurismorrafia, detalhando a técnica cirúrgica utilizada e a evolução pós-operatória. A técnica de rafia simples da parede constitui uma boa alternativa no reparo da parede da FAV quando o aneurisma é de curta extensão, evitando a colocação de um material protético e a confecção de duas anastomoses.
\end{abstract}

Palavras-chave: Derivação arteriovenosa cirúrgica; aneurisma; diálise renal.

\begin{abstract}
One of the most common complications of arteriovenous fistulas (AVF) is aneurysm formation due to weakening of the venous wall after repeated punctures. Its rupture causes severe bleeding that can lead to death. We report the case of a patient with two AVF saccular aneurysms treated by aneurysmorrhaphy. The technique and the postoperative follow-up are presented in detail. Aneurysmorrhaphy is a good option to repair the AVF wall when the aneurysmal segment is short, thus avoiding the placement of prosthetic grafts and the performance of two anastomoses.
\end{abstract}

Keywords: Arteriovenous shunt, surgical; aneurysm; renal dialysis.

\section{Introdução}

Os pacientes com insuficiência renal crônica utilizam como acesso vascular para hemodiálise cateteres ou uma fístula arteriovenosa (FAV). Destes, o acesso ideal é a FAV. A utilização de veia nativa para a confecção da FAV apresenta perviedade superior e menor incidência de complicações quando comparado à prótese ${ }^{1}$. Portanto, é sempre importante prolongar a patência de uma FAV por meio de procedimentos terapêuticos, sejam eles endovasculares ou cirúrgicos convencionais. Dentre as complicações mais frequentes encontradas nas FAVs estão trombose, infecção, aneurisma e estenose. $\mathrm{O}$ aneurisma é definido como uma dilatação fusiforme ou sacular circunscrita com um diâmetro três vezes maior do que o segmento da veia imediatamente a montante e a jusante do sítio de acesso ${ }^{2}$. A formação desses aneurismas geralmente decorre de um enfraquecimento da parede venosa devido às repetidas punções ${ }^{3}$. Na maioria das vezes, o problema é considerado apenas estético e apenas se evita puncionar na área aneurismática ${ }^{3}$. A indicação para correção é quando ocorre dor, infecção, limitação da área de punção, erosão da pele, rápida expansão e baixo fluxo associado à estenose $\mathrm{e}^{1,3}$.

'Cirurgião Vascular da Clínica Vascular de Natal; Professor Auxiliar da Universidade Federal do Rio Grande do Norte (UFRN), Natal (RN), Brasil.

${ }^{2}$ Cirurgião Vascular da Clínica Vascular de Natal, Natal (RN), Brasil.

${ }^{3}$ Médica Nefrologista e Transplantadora Renal da UFRN, Natal (RN), Brasil.

${ }^{4}$ Acadêmica de Medicina da UFRN, Natal (RN), Brasil.

Não foram declarados conflitos de interesse relacionados à publicação deste artigo.

Submetido em: 15.11.2010. Aceito em: 12.04.2011.

J Vasc Bras. 2011;10(2):165-167. 
Diversos procedimentos são atualmente utilizados para corrigir os aneurismas de FAV: aneurismorrafia, ressecção do segmento aneurismático e interposição de prótese, implante de stent-graft e procedimentos combinados.

Descrevemos o caso de um aneurisma de FAV tratado através de aneurismorrafia. O Comitê de Ética em Pesquisa do Hospital Universitário Onofre Lopes da Universidade Federal do Rio Grande do Norte (UFRN) determinou que, para os artigos no formato de relato de caso, é necessário apenas o consentimento informado por escrito do paciente, o qual foi feito neste caso.

\section{Relato do caso}

Paciente com 54 anos, hipertensa, com lúpus e insuficiência renal crônica há 10 anos, em hemodiálise há 5 anos através de uma FAV radiocefálica direita. Há aproximadamente seis meses, notou aumento do diâmetro da FAV próximo ao punho, com dor local associada. O fluxo da FAV durante as sessões de hemodiálise mantinha-se bom, acima de $600 \mathrm{~mL} /$ minuto.

Ao exame físico, apresentava 2 dilatações saculares aneurismáticas da FAV, próximo a anastomose, ambas com aproximadamente $2 \mathrm{~cm}$. A pele sobrejacente aos aneurismas apresentava-se com espessura muito diminuída, com pulsatilidade dos aneurismas bem visível. A FAV tinha bom frêmito.

A paciente foi submetida a bloqueio do plexo braquial. Realizou-se incisão longitudinal diretamente sobre o eixo principal da FAV, evitando-se os aneurismas. Realizou-se também dissecção cuidadosa para liberação dos aneurismas das suas aderências ao subcutâneo para evitar, dessa forma, a rotura destes, pois suas paredes eram extremamente finas, bem como para evitar lesão da pele, que, como mencionamos, tinha espessura muito diminuída.

Após isolamento completo dos 2 aneurismas e de segmentos proximal e distal da FAV para controle, seguiu-se heparinização sistêmica com 5.000 UI de heparina (Figura 1). Realizou-se pinçamento da FAV proximal e distalmente aos aneurismas para interromper o fluxo. Foram colocadas pinças Satinsky na base dos aneurismas junto a segmento normal da parede da FAV, sendo feita a ressecção dos aneurismas.

Os defeitos da parede resultantes da ressecção dos aneurismas foram inspecionados atentamente, e pequenas áreas adicionais de parede aneurismática foram ressecadas (Figura 2). Procedeu-se, então, à rafia, com sutura contínua com polipropileno 6-0. A FAV mostrou-se com bom frêmito e, após rigorosa hemostasia, foi realizada síntese da pele com fio de nylon 4-0 (Figura 3).

A ferida operatória cicatrizou sem intercorrências e, durante esse período, não foi necessário o uso de cateter de hemo-

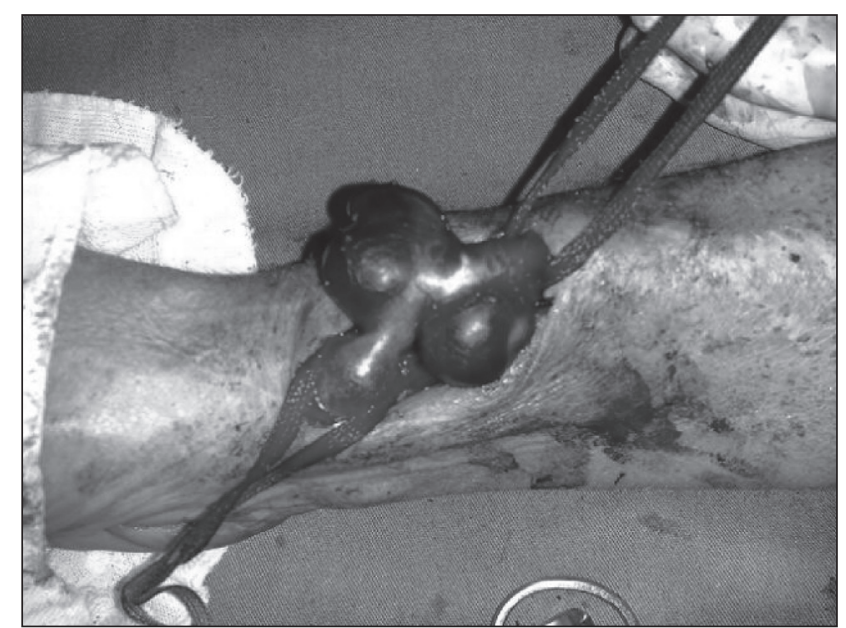

Figura 1. Fístula arteriovenosa radiocefálica direita com presença de dois aneurismas saculares próximos à anastomose.

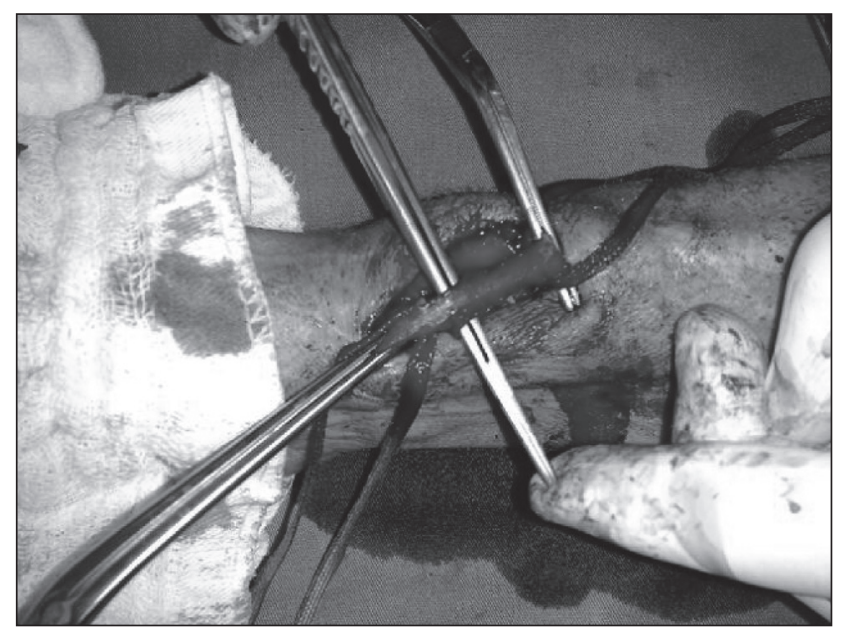

Figura 2. Aspecto após a remoção dos aneurismas. A pinça cirúrgica atravessa o defeito na parede da fístula arteriovenosa resultante da ressecção.

diálise, pois a FAV continuou a ser puncionada nos segmentos mais proximais do antebraço e no braço, fora da área operada.

Após um seguimento de dois anos, a FAV continua sendo utilizada, sem recorrência dos aneurismas no segmento operado e sem o surgimento de outros aneurismas.

\section{Discussão}

A rotura de um aneurisma de FAV causa hemorragia intensa que pode levar à morte. As situações nas quais há mais chance para que isso ocorra são principalmente quando há erosão da pele suprajacente, crescimento rápido do aneurisma e envolvimento da área de anastomose ${ }^{2,3}$.

Atualmente, as principais indicações para a correção dos aneurismas de FAV são seguidas pelas recomendações do KDOQI ${ }^{4}$. 


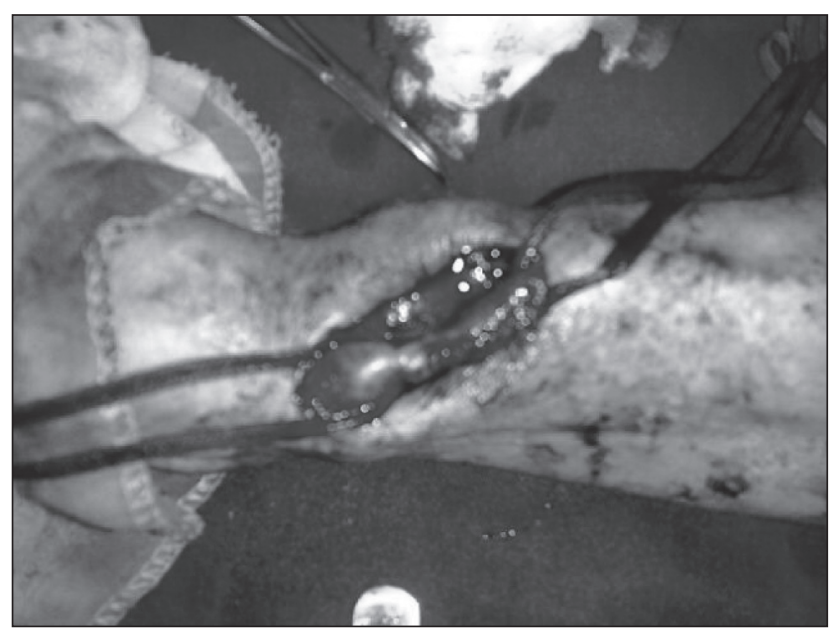

Figura 3. Aspecto final após a correção com a técnica de aneurismorrafia.

Nossa paciente possuía um aneurisma de FAV com dor local e início de sofrimento da pele suprajacente além da proximidade da área da anastomose.

Um dos tratamentos propostos para correção constituise na plicatura do "excesso" de parede, de modo que nenhuma ressecção é necessária, segundo Lo e Tan ${ }^{5}$.

A revisão cirúrgica de pseudoaneurisma e de um verdadeiro aneurisma de FAV apresenta patência primária pósintervenção aceitável e, portanto, é justificada, de acordo com Georgiadis et al. ${ }^{6}$. Ainda segundo esses autores, os melhores resultados da correção são encontrados em aneurisma de FAV com veia nativa, aneurisma verdadeiro, aneurismas em antebraço e na presença de um ou dois aneurismas.

O tratamento também pode ser feito com o implante de stent-graft, mas geralmente este é reservado para a correção de pseudoaneurisma e não aneurisma verdadeiro e, principalmente, em FAV com prótese ${ }^{7,8}$.

O revestimento externo da veia com material prostético após a aneurismorrafia também pode ser utilizado e, segundo Balaz et al. ${ }^{9}$, pode diminuir a hiperplasia intimal.

A aneurismorrafia pode ser feita também ressecando-se a pele adjacente, principalmente se esta estiver lesada. Nos casos com estenose associada ao aneurisma, o segmento afetado pode ser ressecado, fazendo-se uma reconstrução com anastomose término-terminal ${ }^{2}$.

Procedimentos utilizando técnica endovascular e cirúrgica convencional também podem ser utilizados, conforme exemplo de Kouvelos et al. ${ }^{10}$, para o tratamento de FAV com prótese na coxa com aneurisma e estenose associada.

Neste caso, o aneurisma apresentava uma dilatação sacular de cada lado da FAV por uma extensão relativamente curta. Após a ressecção da parede aneurismática, o bom aspecto macroscópico da parede venosa restante e a curta extensão necessária para uma rafia fez-nos optar pela simples rafia contínua das paredes laterais da FAV. Nossa segunda opção seria ressecar todo o segmento lesado e interpor um segmento de prótese.

A rafia simples da parede constitui uma boa alternativa na ressecção de aneurismas de curta extensão, evitando a colocação de um material protético e a confecção de duas anastomoses.

\section{Referências}

1. National Kidney Foundation [Internet]. The National Kidney Foundation Kidney Disease Outcomes Quality Initiative (NKF KDOQI ${ }^{\text {mm}}$ ) [citado 2011 abr 23]. http://www.kidney.org/ professionals/kdoqi.

2. Berard X, Brizzi V, Mayeux S, et al. Salvage treatment for venous aneurysm complicating vascular access arteriovenous fistula: use of an exoprosthesis to reinforce the vein after aneurysmorrhaphy. Eur J Vasc Endovasc Surg. 2010;40:100-6.

3. Schanzer $\mathrm{H}$. Overview of complications and management after vascular access creation. In: Gray RJ, Sands JJ, editors. Dialysis access: a multidisciplinary approach. Philadelphia: Lippincott Williams \& Wilkins; 2002. p. 93-7.

4. National Kidney Foundation [Internet]. NKF-DOQI Clinical Practice Guidelines for Vascular Access. Guideline 5 - Treatment of fistula complications [citado 2011 mai 2]. http://www.kidney.org/ professionals/KDOQI/guideline_upHD_PD_VA/va_guide5.htm

5. Lo HY, Tan SG. Arteriovenous fistula aneurysm--plicate, not ligate. Ann Acad Med Singapore. 2007;36:851-3.

6. Georgiadis GS, Lazarides MK, Panagoutsos SA, et al. Surgical revision of complicated false and true vascular access-related aneurysms. J Vasc Surg. 2008;47:1284-91.

7. Ananthakrishnan G, Bhat R, Severn A, Chakraverty S. Stent graft exclusion of pseudo-aneurysm arising from PTFE hemodialysis graft after recurrence following ultrasound guided thrombin injection. J Vasc Access. 2008;9:293-5.

8. Criado E, Marston WA, Ligush J, Mauro MA, Keagy BA. Endovascular repair of peripheral aneurysms, pseudoaneurysms and arteriovenous fistulas. Ann Vasc Surg. 1997;11:256-63.

9. Balaz P, Rokosny S, Klein D, Adamec M. Aneurysmorrhaphy is an easy technique for arteriovenous fistula salvage. J Vasc Access. 2008;9:81-4.

10. Kouvelos GN, Xanthopoulos DK, Harissis HV, Arnaoutoglou HM, Matsagas MI. Hybrid management of a false aneurysm complicating an arteriovenous graft in a patient with critical limb ischemia. J Vasc Access. 2009;10:216-8.

$$
\begin{array}{r}
\text { Correspondência: } \\
\text { Ricardo Wagner da Costa Moreira } \\
\text { Avenida Campos Sales, 759, apto 800 - Tirol } \\
\text { CEP 59020-300 - Natal (RN), Brasil } \\
\text { E-mail: ricardowcm@yahoo.com.br } \\
\text { Contribuições dos autores: } \\
\text { Concepção e desenho do estudo: RWCM } \\
\text { Análise e interpretação dos dados: RWCM, DDRC } \\
\text { Coleta de dados: KMAHC, RBBP } \\
\text { Redação do artigo: RWCM, RBBP } \\
\text { Revisão crítica do texto: KMAHC, DDRC } \\
\text { Análise estatística: RWCM } \\
\text { Aprovação final do artigo*: RWCM, RDRC, KMAHC, RBBP } \\
\text { Responsabilidade geral pelo estudo: RWCM } \\
\text { Informações sobre financiamento: N/A. }
\end{array}
$$
*Todos os autores leram e aprovaram a versão final submetida ao J Vasc Bras. 